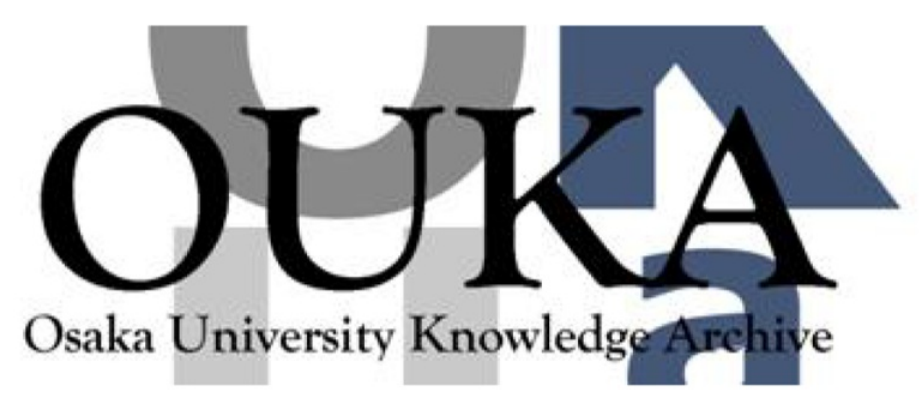

\begin{tabular}{|c|l|}
\hline Title & $\begin{array}{l}\text { Low-temperature elastic and piezoelectric } \\
\text { constants of paratellurite }(\alpha-\operatorname{Te} 02)\end{array}$ \\
\hline Author(s) & $\begin{array}{l}\text { Ledbetter, Hassel; Leisure, Robert G. ; } \\
\text { Migliori, Albert et al. }\end{array}$ \\
\hline Citation & $\begin{array}{l}\text { Journal of Applied Physics. 96(11) p. 6201- } \\
\text { p. } 6206\end{array}$ \\
\hline Issue Date & $2004-11-22$ \\
\hline oaire:version & VoR \\
\hline URL & $\begin{array}{l}\text { https://hdl. handle. net/11094/84222 } \\
\text { This article may be down loaded for personal use } \\
\text { only. Any other use requires prior permission } \\
\text { of the author and AIP Publishing. This article } \\
\text { appeared in Journal of Applied Physics, 96(11), } \\
6201-6206 \text { (2004) and may be found at } \\
\text { https://doi.org/10.1063/1.1805717. }\end{array}$ \\
\hline rights \\
\hline Note & \begin{tabular}{l}
$\mid$ \\
\hline
\end{tabular} \\
\hline
\end{tabular}

Osaka University Knowledge Archive : OUKA

https://ir. Library. osaka-u. ac. jp/

0saka University 


\title{
Low-temperature elastic and piezoelectric constants of paratellurite $\left(\alpha-\mathrm{TeO}_{2}\right)$
}

\author{
Hassel Ledbetter ${ }^{a)}$ \\ Los Alamos National Laboratory, Los Alamos, New Mexico 87545 \\ Robert G. Leisure \\ Physics Department, Colorado State University, Fort Collins, Colorado 80523 \\ Albert Migliori and Jon Betts \\ Los Alamos National Laboratory, Los Alamos, New Mexico 87545 \\ Hirotsugu Ogi \\ Graduate School of Engineering Science, Osaka University, Osaka 560-8531, Japan
}

(Received 16 March 2004; accepted 17 August 2004)

\begin{abstract}
We report paratellurite's 300-10-K elastic constants $C_{i j}$, six constants reflecting tetragonal symmetry (P422 point group). The quantity $e_{14}^{2} / k_{11}$, where $e_{14}$ denotes the sole independent piezoelectric constant and $k_{11}$ denotes a dielectric constant, was determined over the same temperature range. All the principal $C_{i j}$ show approximately regular temperature-change behavior: increasing with decreasing temperature, the increases being $2 \%-10 \%$. One derived elastic constant, $C^{\prime}=\left(C_{11}\right.$ $\left.-C_{12}\right) / 2$, a shear constant, shows strongly anomalous temperature behavior, decreasing continuously during cooling to $10 \mathrm{~K}$, the total decrease being $5 \%$. The quantity $e_{14}^{2} / k_{11}$ was essentially independent of temperature. Obtained by resonant-ultrasound spectroscopy, our results differ from previous studies, none of which went to $10 \mathrm{~K}$, a temperature region of much practical interest for this material. From the near-zero-temperature elastic constants, we derive a Debye temperature of $235 \mathrm{~K}$. The slope $d C^{\prime} / d T$ yields a negative Gruneisen parameter $\gamma=-0.9$, close to the specific-heat value. (C) 2004 American Institute of Physics. [DOI: 10.1063/1.1805717]
\end{abstract}

\section{INTRODUCTION}

Recently, ${ }^{1}$ two of the present authors presented an extensive study of paratellurite's ambient-temperature elastic, internal-friction, and piezoelectric coefficients. The present study represents an extension to low temperatures for the elastic and piezoelectric constants. Just as the previous study helped clarify the ambient-temperature $C_{\mathrm{ij}}$, the present study clarifies the disparate previously reported $d C_{i j} / d T,{ }^{2-5}$ extends the measurements to $10 \mathrm{~K}$, and yields insights into paratellurite's remarkable elastic properties.

Previously, ${ }^{1}$ we described much of paratellurite's odd elastic behavior. Some remarkable aspects include the following.

(1) Strong shear-wave anisotropy: $C_{66} / C^{\prime}=281$ ! This property led to paratellurite being chosen to confirm Herring's anharmonic phonon-relaxation theory. ${ }^{2}$ The high anisotropy ratio reflects a low $C^{\prime}$. Related to this, there remains the following question: reflecting a very low $C^{\prime}$, does paratellurite undergo a low-temperature phase transformation caused by decreased volume?

(2) Along the $x$ axis, one shear-wave velocity exceeds the longitudinal-wave velocity: $C_{66}>C_{11}$. Isotropic materials show shear-wave velocities approximately half the longitudinal-wave velocity.

(3) The principal-axis Poisson ratios vary widely, from 0.02 to 0.91 .

${ }^{a)}$ Electronic mail: hledbet@lanl.gov
(4) The [110] [001] Poisson ratio is negative.

(5) For an oxide, the elastic constants are low, especially when compared with a companion-crystal-structure material: rutile $\left(\mathrm{TiO}_{2}\right)$.

As discussed previously, most of paratellurite's remarkable elastic behavior can be understood from its crystal structure and interatomic bonding, which also determine its unusual optical properties. Key features here include alternating weak and strong bonds in the Te-O chains and a Te-O truss structure that is soft against several mechanical deformations.

Paratellurite permits unusual piezoelectric applications because its only piezoelectric coefficient is a shear coefficient $e_{14}$. Thus, it generates and detects only shear waves.

Interest in paratellurite's low-temperature properties arises for several reasons.

(1) Often, piezoelectric crystals show irregular lowtemperature physical-property changes.

(2) Previous studies found a strong decrease in $C^{\prime}$ upon cooling, but the zero-temperature value could not be determined reliably by extrapolation. Also, the value of $C^{\prime}$ is important for considering phase stability.

(3) At low temperatures, paratellurite shows a negative overall (average-over-direction) Gruneisen parameter, which relates to elastic-constant temperature derivatives $d C_{i j} / d T$.

(4) Paratellurite sees important low-temperature uses, for example, in the massive (eventually tons) underground 
cryogenic bolometer detector for sensing rare events (related to dark matter). ${ }^{6}$ For this particular detector, one uses the Debye temperature to predict the bolometer's pulse amplitude for a given energy deposition. The main error in estimating paratellurite's Debye temperature arises from the difficulty of extrapolating its $C_{i j}$ to zero temperature. Especially, $C^{\prime}$ exacerbates the extrapolation: $C^{\prime}$ is small, its temperature derivative is large, and small shear elastic constants contribute strongly to the Debye temperature. $^{7}$

(5) Finally, because paratellurite represents one of the most oddly anisotropic materials yet studied, one wonders whether the elastic anisotropy depends strongly on temperature, that is, whether the anisotropy is intrinsic or is temperature induced.

\section{MEASUREMENTS}

Our crystal was a companion to that used in the previous ambient-temperature measurments. ${ }^{1}$ It consisted of an accurate parallelepiped with faces perpendicular to the tetragonal $a, b, c$ axes as confirmed by Laue X-ray diffraction within $0.2^{\circ}$. The corresponding room-temperature dimensions were $7.030,8.035$, and $8.999 \mathrm{~mm}$. The room- temperature specific gravity, calculated from the dimensions and mass, was 5.990. This is just below the x-ray-diffraction value, 6.023. We measured the elastic moduli using resonant ultrasound spectroscopy (RUS). ${ }^{8-10}$ To make measurements between 300 and $10 \mathrm{~K}$, we used a gas-flow cryostat.

\section{RESULTS}

As mentioned above, $\mathrm{TeO}_{2}$ is piezoelectric; this affects the specimen's resonance frequencies. Thus, one must modify the usual inverse calculation ${ }^{10}$ to consider the piezoelectric effect. ${ }^{11}$ With the inclusion of the piezoelectric effect in the Langrangean-minimization method, it is possible to determine both the elastic constants and the piezoelectric constants from the measured frequencies. ${ }^{1,12}$ For the present study, the frequencies of 38 of the lowest 40 resonances were fit with an rms difference between measured and computed frequencies that ranged from $0.13 \%$ at room temperature to a maximum of $0.23 \%$ at lowest temperature. Specimen dimensions were corrected for temperature dependence using reported thermal-expansion measurements. ${ }^{13}$

Paratellurite has tetragonal symmetry (P422 point group) with six independent elastic constants: $C_{11}, C_{33}, C_{44}, C_{66}$, $C_{12}, C_{13}$. Figure 1 shows $C_{11}, C_{12}$, and $C_{66}$-as well as the bulk modulus $B$-as a function of temperature. Figure 2 shows $C_{33}$ along with moduli derived from the principal $C_{i j}$ to be discussed below. Finally, Fig. 3 shows $C_{44}$ determined without including the piezoelectric effect in the minimization scheme, as well as this modulus determined by including the piezoelectric effect: $C_{44}$ (nonpiezo) and $C_{44}$ (piezo). Because the only independent piezoelectric coefficient is $e_{14}$, the only elastic constant affected by the piezoelectricity is $C_{44}$. Figure 3 shows that the effect of ignoring the piezoelectric effect is to increase the apparent $C_{44}$ by about $0.75 \mathrm{GPa}$. Calculations with and without including the piezoelectric effect for the

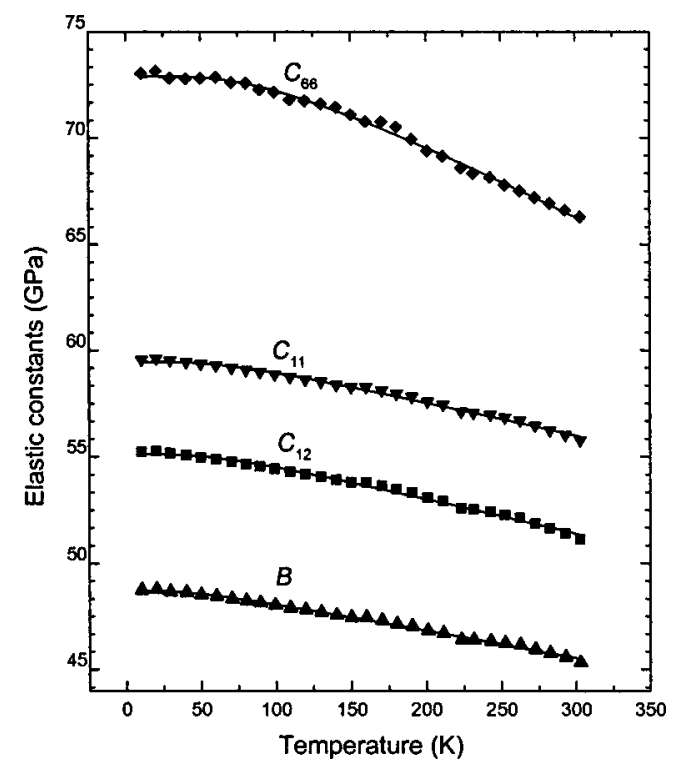

FIG. 1. Low-temperature elastic constants $C_{11}, C_{12}, C_{66}$ and the bulk modulus $B$. Points represent measurements. Curves represent Einstein-oscillator behavior, Eq. (2).

other principal $C_{i j}$ gave essentially the same result; the other $C_{i j}$ were not affected by the piezoelectricity. Curves through the measurement points represent an Einstein-oscillator model based on the idea that elastic stiffness varies as ${ }^{14}$

$$
C(T)=C(0)(1-D \bar{E}) .
$$

Here, $C(0)$ denotes the zero-temperature elastic stiffness, $\bar{E}$ the average oscillator energy, and $D$ a constant that depends on crystal structure. Substituting the usual expression for the Einstein-oscillator energy gives ${ }^{15}$

$$
C(T)=C(0)-\frac{s}{\exp [t / T]-1} .
$$

Here, $t$ relates to the Einstein temperature, but is usually lower because, in most crystals, the average phonon fre-

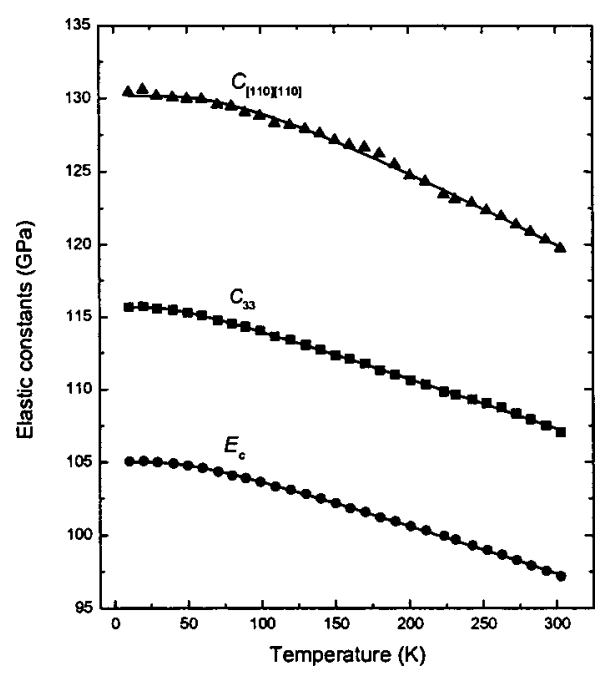

FIG. 2. Low-temperature elastic constants $C_{33}, C_{[110][110]}$ (corresponding to longitudinal waves along [110] ), and the Young modulus $E_{c}$ (corresponding to extension-compression along [001] ). Points represent measurements. Curves represent Einstein-oscillator behavior, Eq. (2). 


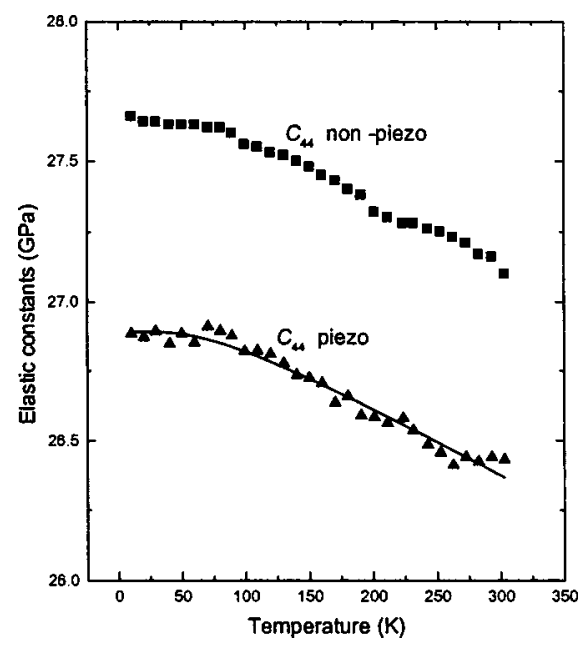

FIG. 3. Low-temperature elastic constants of $C_{44}$. The points for $C_{44}$ nonpiezo were determined with the Langrangean minimization method without taking the piezoelectric effect into account while those for $C_{44}$ piezo were determined by including the piezoelectric effect in the minimization. The points represent measurements. The curve through the $C_{44}$ piezo data represents an Einstein-oscillator behavior, Eq. (2).

quency $\bar{\omega}$ is lower than the Einstein frequency $\omega_{E}$. Originally, $s$ was an adjustable parameter. Later, a relationship appeared between $s$ and three basic properties: Einstein temperature, Gruneisen parameter, and atomic volume. ${ }^{16}$ The fit parameters $C(0), s$, and $t$, for the six principal $C_{i j}$ are given in Table I. All six constants show a normal temperature dependence, increasing as the temperature decreases. Except for $C_{44}$ the increase ranges from $7 \%$ to $10 \%$. The increase for $C_{44}$ is small, less than $2 \%$.

Figure 4 shows $e_{14}^{2} / k_{11}$ where $e_{14}$ denotes the sole independent piezoelectric constant for the symmetry of $\mathrm{TeO}_{2}$ and $k_{11}$ denotes a dielectric constant. The fitting of the material parameters to the measured frequencies gives this combination of the piezoelectric constant and the dielectric constant, not each separately. As the figure shows, this quantity remains essentially constant at about $0.80 \mathrm{GPa}$ over the entire studied temperature range, $10-300 \mathrm{~K}$.

It is useful to consider other elastic parameters that can be calculated from the six Voigt $C_{i j}$. Thus, Fig. 1 shows the bulk modulus $B$ along with a fit of Eq. (2). The temperature dependence is normal, increasing about $8 \%$ as the temperature decreases to $10 \mathrm{~K}$. Figure 2 includes Young's modulus $E_{c}$ representing extension resistance along the tetragonal $c$ axis. Also included in Fig. 2 is the elastic constant

TABLE I. Parameters obtained by fitting Eq. (2) to measurements.

\begin{tabular}{lccc}
\hline \hline Modulus & $C(0)(\mathrm{GPa})$ & $s(\mathrm{GPa})$ & $t(\mathrm{~K})$ \\
\hline$C_{11}$ & 59.5 & 3.08 & 191 \\
$C_{12}$ & 55.1 & 3.01 & 178 \\
$C_{13}$ & 24.7 & 0.47 & 69 \\
$C_{33}$ & 115.6 & 4.51 & 129 \\
$C_{44}$ & 26.9 & 0.53 & 212 \\
$C_{66}$ & 72.9 & 10.0 & 277 \\
$B$ & 48.7 & 1.70 & 129 \\
$E_{c}$ & 105.0 & 5.29 & 158 \\
$C_{[110][110]}$ & 130.1 & 13.1 & 248 \\
\hline \hline
\end{tabular}

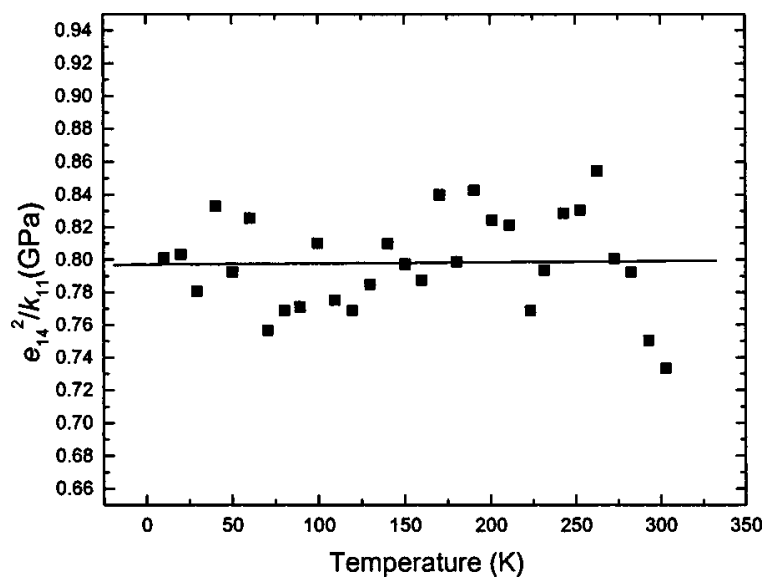

FIG. 4. The quantity $e_{14}^{2} / k_{11}$ vs temperature, where $e_{14}$ denotes the piezoelectric constant and $k_{11}$ a dielectric constant. The piezoelectric effect increases $C_{44}$ by $3 \%$.

$$
C_{[110][110]}=\frac{C_{11}+C_{12}+2 C_{66}}{2},
$$

which corresponds to propagation of longitudinal elastic waves in the [110] direction. Figure 5 shows Young's modulus for extension along the tetragonal $a$ ( or $b$ ) axis as well as the elastic constant

$$
C^{\prime}=\frac{C_{11}-C_{12}}{2},
$$

the elastic constant corresponding to shear waves propagated along [110] and polarized along [110]. Figure 5 shows large differences from the previous figures. First, the temperature dependence is opposite; these constants decrease with decreasing temperature. There are other differences, several of which were discussed previously. ${ }^{1} C_{11}$ ranges from $84 \%$ to $81 \%$ of $C_{66}$ as the temperature is lowered from room temperature to $10 \mathrm{~K}$, meaning that for acoustic waves along

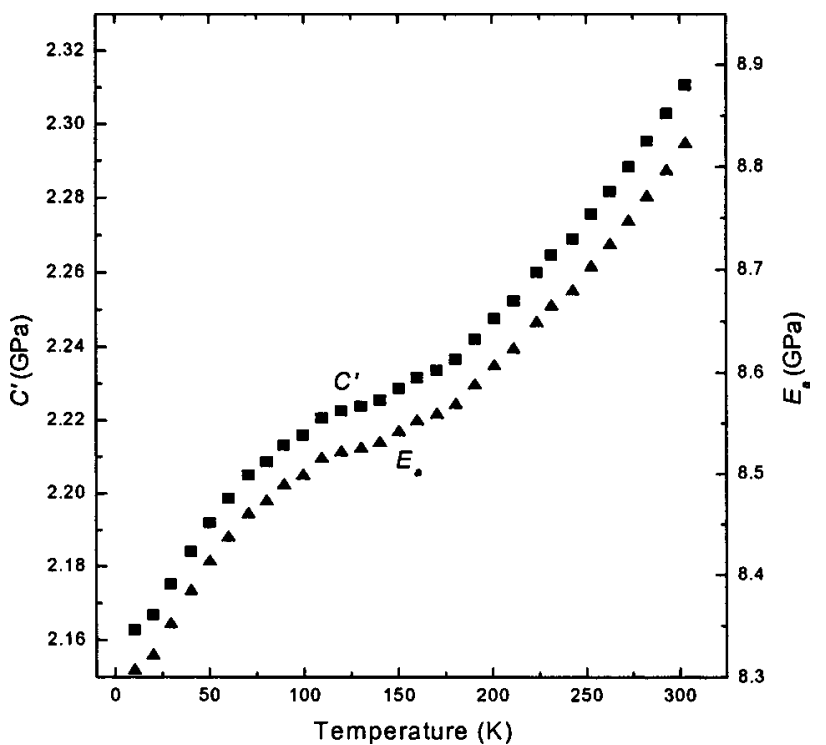

FIG. 5. Temperature dependence of the elastic constant $C^{\prime}$, corresponding to shear on a (110) plane in a [ $\overline{1} 10]$ direction, and the Young modulus $E_{a}$, corresponding to extension-compression along the crystalline $a$ axis. 


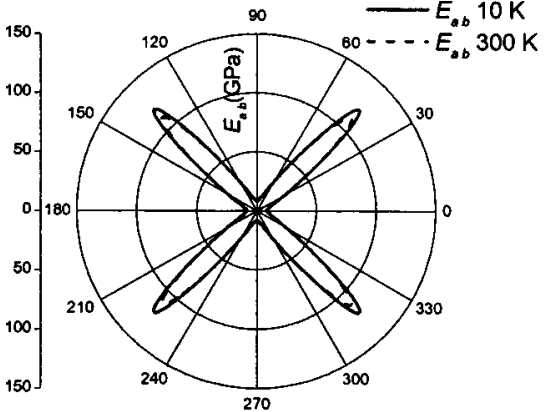

(a)

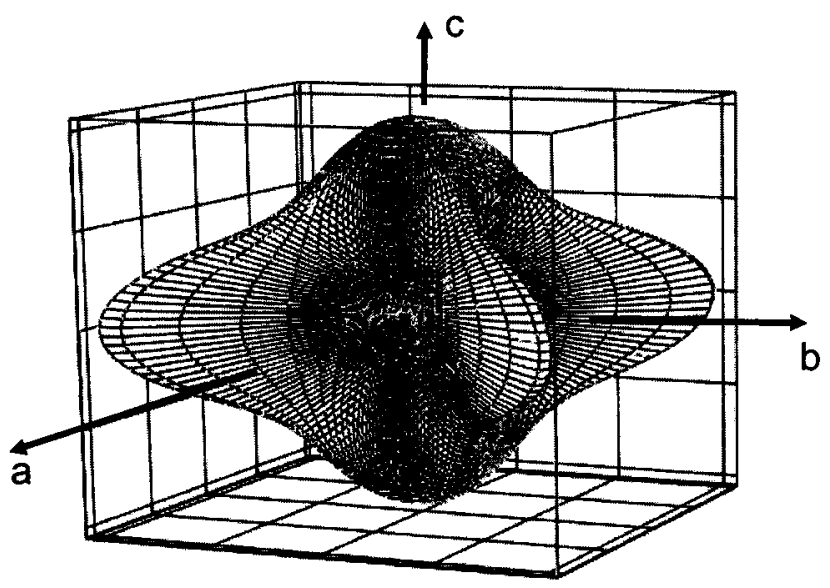

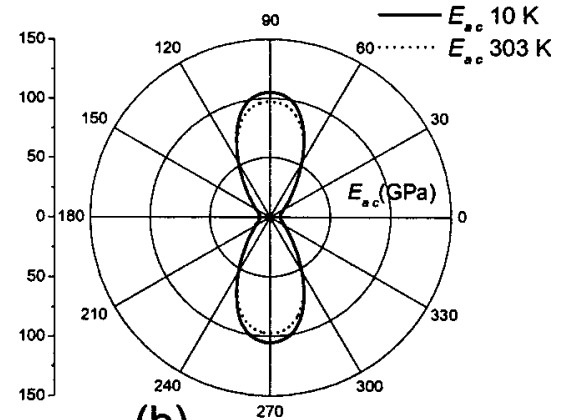

(b)

FIG. 6. Directional dependence of the Young modulus. (a) Modulus in the crystalline $a-b$ plane as a function of the angle from the $a$ axis. (b) Modulus in the $a-c$ plane as a function of the angle from the $a$ axis. (c) Threedimensional representation of the Young modulus at $10 \mathrm{~K}$.

(c)

[100] the longitudinal wave is slower that the shear wave polarized along [010]. The situation changes dramatically upon rotating $45^{\circ}$ to the [110] direction. In this case the elastic constant $C_{[110][110]}$ ranges from 52 to 60 times $C^{\prime}$ as the temperature decreases. The shear wave corresponding to $C^{\prime}$ is extraordinarily slow.

The strong elastic anisotropy is revealed further by considering the directional dependence of the Young modulus. Figure 6(a) shows the strong anisotropy in the crystalline $a-b$ plane where the modulus increases by a factor of 14.7 at $10 \mathrm{~K}$ on rotating $45^{\circ}$ from the [100] to the [110] direction. Similarly, Fig. 6(b) shows the anisotropy in the $a-c$ plane, where $E_{c} / E_{a}=12.7$ at $10 \mathrm{~K}$. Finally, Fig. 6(c) presents a three-dimensional plot of the Young modulus at $10 \mathrm{~K}$.

We gain further insight by considering the Poisson ratio

$$
\nu_{i j}=-\frac{\varepsilon_{j}^{\prime}}{\varepsilon_{i}^{\prime}} .
$$

Here, $\varepsilon_{i}^{\prime}$ denotes the longitudinal strain in response to a longitudinal stress along the $x_{i}^{\prime}$ axis and $\varepsilon_{j}^{\prime}$ the corresponding lateral strain along the $x_{j}^{\prime}$ axis. We consider three cases, where the prime coordinates refer to rotations about the crystalline $a, b, c$ axes. ${ }^{17}$ Figure 7 shows the Poisson ratios $v_{12}^{\prime}$ and $v_{13}^{\prime}$ for rotation about the crystalline $a$ axis. The longitudinal strain is along the $x_{1}^{\prime}$ axis and the lateral strains are along the $x_{2}^{\prime}$ and $x_{3}^{\prime}$ axes. For $\theta=0, \nu_{12}^{\prime}$ and $\nu_{13}^{\prime}$ reduce to the usual $\nu_{12}$ and $\nu_{13}$. Because of symmetry, the diagram is symmetric about the $45^{\circ}$ angle. Figure 8 shows a similar diagram for rotation about the crystalline $b$ axis. Rotation about $b$ is not equivalent to rotation about $a$ because the longitudinal extension is along $x_{1}^{\prime}$, which is not equivalent for the two cases. Figure 8 shows the remarkable result that a longitudinal extension in the $a-c$ plane at about $60^{\circ}$ above the $a$ axis results in a contraction along the $x_{2}^{\prime}$ axis of magnitude 1.54 as great. Also, $\nu_{13}^{\prime}$ is negative for this orientation, reaching a value of -0.845 at $60.9^{\circ}$. Such a large negative Poisson ratio is unusual. This means a lateral expansion that is nearly as large as the longitudinal expansion. Negative Poisson ratios are unusual, but not unknown. Recently, many negative-

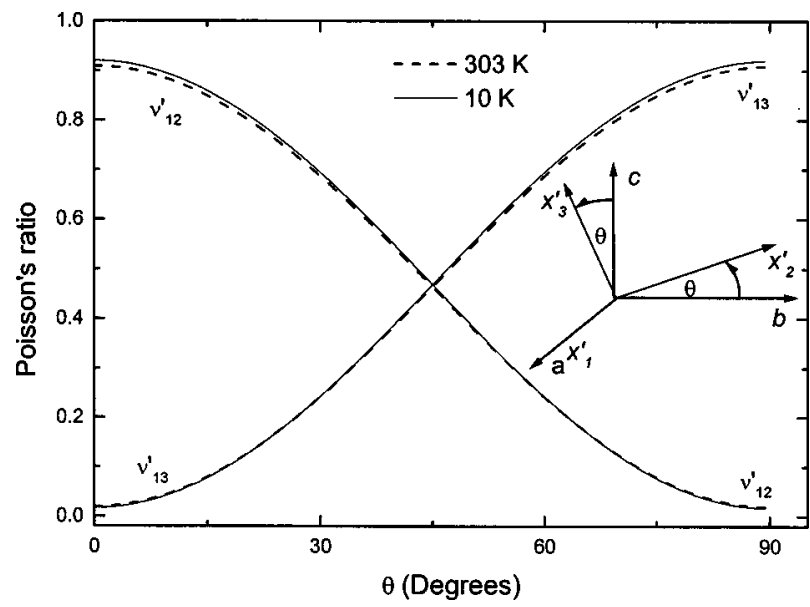

FIG. 7. Poisson ratios describing the response to a longitudinal stress along the $x_{1}^{\prime}$ axis as a function of the angle of rotation $\boldsymbol{\theta}$ about the $a$ axis. A positive value of $\nu_{12}^{\prime}\left(\nu_{13}^{\prime}\right)$ corresponds to the contraction along the $x_{2}^{\prime}\left(x_{3}^{\prime}\right)$ axis in response to an extension along $x_{1}^{\prime}$. 


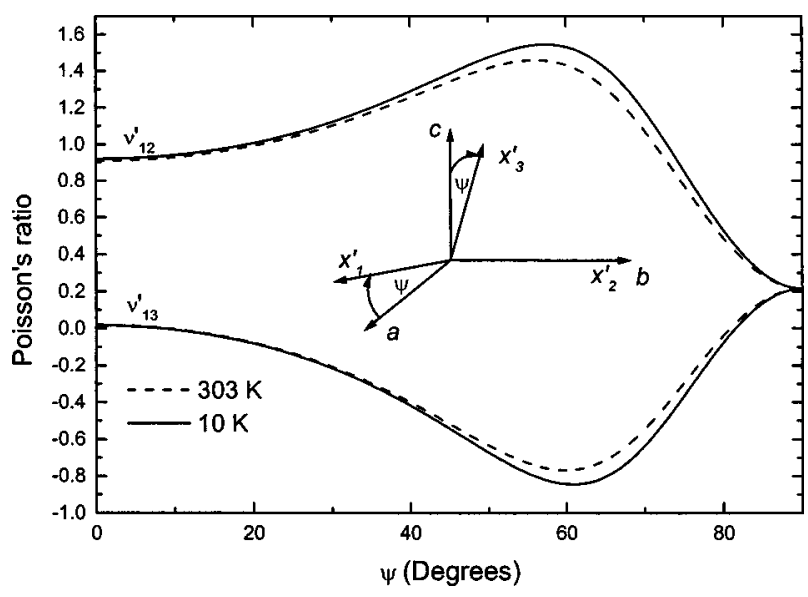

FIG. 8. Poisson ratios describing the response to a longitudinal stress along the $x_{1}^{\prime}$ axis as a function of the angle of rotation $\psi$ about the $b$ axis. The negative value of $\nu_{13}^{\prime}$ means there is an extension along the $x_{3}^{\prime}$ axis in response to an extension along $x_{1}^{\prime}$.

Poisson-ratio studies appeared. ${ }^{18-21}$ Finally, Fig. 9 gives the Poisson ratios for rotation of the primed system about the crystalline $c$ axis. As pointed out previously, ${ }^{1}$ for this case $\nu_{12}^{\prime}$ becomes negative for a longitudinal extension along the [110] axis.

Table I gives the parameters from fitting Eq. (2) to those elastic constants having a normal temperature dependence. These parameters permit a convenient calculation of the elastic constants as functions of temperature.

\section{DISCUSSION}

All six principal Voigt elastic constants $-C_{11}, C_{33}, C_{44}$, $C_{66}, C_{12}, C_{13}$-show nominally regular temperature behavior. In accord with Eq. (2), they show linearity at higher temperatures, increases upon cooling, zero slopes approaching zero temperature. Regular behavior also appears in the derived elastic constants $B, E_{c}$, and $C_{[110][110]}$. The exceptionally low $C^{\prime}$ value, discussed in more detail below, resulted in many of the lower resonance frequencies depending strongly on this elastic constant. There was only a weak dependence of the frequencies on the elastic constants $C_{13}$ and $C_{66}$, leading to more scatter in the results for these two constants. A

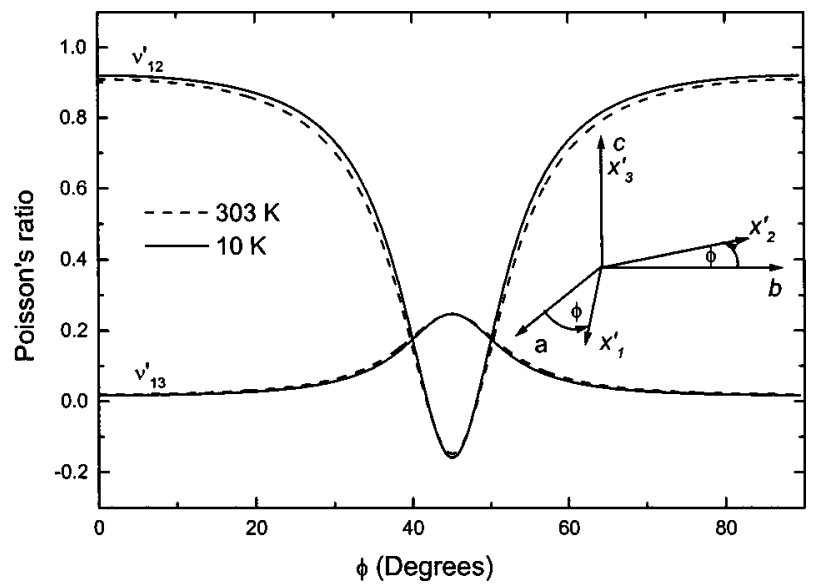

FIG. 9. Poisson ratios describing the response to a longitudinal stress along the $x_{1}^{\prime}$ axis as a function of the angle of rotation $\phi$ about the $c$ axis. derived shear-mode elastic constant, $C^{\prime}=\left(C_{11}-C_{12}\right) / 2$, shows dramatically different temperature behavior. Upon cooling, it decreases approximately linearly, about 5\%. $C^{\prime}$ represents resistance to shear on (110) in $[\overline{1} 10] . C^{\prime}$ is remarkably small, about $4 \%$ and $9 \%$ of the principal shear moduli $C_{44}$ and $C_{66}$. Such a small shear modulus reflects a soft-phonon mode, often indicating incipient phase transformation. ${ }^{22}$ The $C^{\prime}$ decrease during cooling reflects increased tendency toward mechanical instability. Paratellurite represents a rare material where one elastic constant changes with temperature so drastically from all the other principal elastic constants. Although a phase transformation in paratellurite fails to result from decreasing temperature, a reversible second-order transformation occurs under a small pressure $(9$ kbar). ${ }^{23}$ Stable phases depend strongly on the average atomic volume. Using reported thermal-expansion coefficients, ${ }^{13}$ we estimate the $300-0-\mathrm{K}$ volume change $\Delta V / V$ as 0.0083 . From Hooke's law and a bulk modulus of $45.3 \mathrm{GPa}$, we estimate the pressure-induced volume change as 0.020 , about 2.5 times the volume change effected by cooling.

Paratellurite's much smaller thermal-expansion coefficient along the $c$-axis compared with the $a$-axis ${ }^{13}$ suggests smaller temperature-induced changes in $C_{33}$ than in $C_{11}$, contrary to observation. This disagreement could be explained by atomic-position changes in the unit cell during cooling. Above, we mentioned unusual Poisson ratios and some soft Te-O interatomic bonds. Our results show that the principal Poisson ratios given by $\nu_{i j}=-S_{i j} / S_{i i}$, despite their unusual values, change surprisingly little during cooling. (Here $S_{i j}$ denotes the tensor inverse of $C_{i j}$.) Atomic-position changes are likely because it was suggested that paratellurite's low $C^{\prime}$ value arises because internal atomic displacements occur in response to a homogeneous mechanical $C^{\prime}$-mode strain. ${ }^{24}$

Focusing on $d C^{\prime} / d T$, we found a concave slope in the region $150-300 \mathrm{~K}$, agreeing with a previous measurement, ${ }^{5}$ but disagreeing with another that reported a convex shape. ${ }^{2}$ The slope relates to the mode Gruneisen parameter. For the bulk modulus $B$, at higher temperatures (say above half the Debye temperature), in a quasiharmonic model, ${ }^{16}$

$$
\frac{d B}{d T}=-\frac{3 k \gamma(\gamma+1)}{V_{a}} .
$$

Here, $k$ denotes Boltzmann constant, $\gamma$ Gruneisen parameter, $V_{a}$ atomic volume. Although it remains to be derived, a similar expression should result for each elastic constant. Equation (6) and the strong positive $d C^{\prime} / d T$ suggests strongly that, even at ambient temperatures, the mode Gruneisen parameter corresponding to $C^{\prime}$ must be negative. Substitution into the $C^{\prime}$ form of Eq. (6), taking the negative root, yields $\gamma=-0.9$. A negative mode Gruneisen parameter is implied also by the pressure measurements that showed a negative $d C^{\prime} / d P{ }^{23}$ Taking the approximate relationship ${ }^{25}$

$$
\frac{d C_{i}}{d P}=2 \gamma_{i}+1
$$

yields $\gamma_{i}=-1.8$, near the value -1.4 estimated by White and co-workers ${ }^{13}$ from other researchers' pressure measurements, 
and close to their overall value $\gamma=-1$ at very low temperatures where $C^{\prime}$ dominates the specific heat.

Although the six principal $C_{i j}$ show nearly normal temperature dependencies, some derived elastic constants, notably $\left(C_{11}-C_{12}\right) / 2$ and $E_{a}=1 / S_{11}$ (Fig. 5), show abnormal stiffening near $150 \mathrm{~K}$. Not shown here, abnormal changes occur also in the elastic compliances $S_{11}$ and $S_{12}$. No irregularities appeared in $S_{33}, S_{44}, \mathrm{~S}_{66}$. Thus, the irregularity appears only in those principal $S_{i j}$ corresponding to a strain along the $x_{1}$ or $x_{2}$ axes. This irregularity's atomistic cause remains uncertain.

Now, we consider the Debye temperature $\Theta_{D}$. Specificheat measurements yielded $265 \mathrm{~K} .{ }^{13}$ The same authors, from extrapolated elastic-constant measurements, estimated 240 K. Recent high-precision specific-heat measurements to $0.06 \mathrm{~K}$ yielded $232 \mathrm{~K}$. ${ }^{6}$ Our previous ambient-temperature elastic-constant measurements yielded $232 \mathrm{~K}$. Using our present measurements extrapolated to zero temperature, we obtain $235 \mathrm{~K}$. The surprisingly small difference from our ambient-temperature $C_{i j}$ estimate arises from the strong effect of the $C^{\prime}$ decrease offsetting the increases in all the principal six $C_{i j}$. The unusually small $C^{\prime}$ means that this elastic-vibration mode dominates the Debye temperature and the low-temperature specific heat.

Related to mode Debye temperatures are the $t$ coefficients shown in Table I, coefficients obtained from fitting Eq. (2) to the measurements. In an Einstein solid, $t=\Theta_{E}$, where $\Theta_{E}$ denotes Einstein temperature. In real solids, usually $t<\Theta_{E}$ because the average phonon frequency is usually less than the Einstein frequency. In Table I, several $t$ values exceed the Einstein temperature. (Here, $\Theta_{E}=0.75 \Theta_{D}=176 \mathrm{~K}$.) The surprising higher $t$ values for both longitudinal and shear modes suggest higher-frequency-phonon modes in the phonon density of states. The notable softness of $C_{13}(T)$ presents interpretation problems because $C_{13}$ is nonphysical, that is, the lattice contains no vibrations (standing waves) related only to $C_{13}$; likewise for $C_{12}$.

Finally, we comment on the interatomic bonding. Some authors concluded that paratellurite possesses covalent bonding, reflecting the double (four-electron) bond in the $\mathrm{TeO}_{2}$ molecule. $^{26}$ But, piezoelectricity requires ionic bonding. ${ }^{27}$ Several attempts to relate interatomic-bonding type to the $C_{i j}$ appeared previously. ${ }^{28-30}$ Paratellurite's complicated crystal structure seems to preclude those approaches. Considering all the $C_{i j}$ and their usual combinations, the best indicators of paratellurite's interatomic-bonding type are its negative Poisson ratios. Very small Poisson ratios occur in some covalent crystals, but apparently never in ionic crystals.

\section{CONCLUSIONS}

(1) For paratellurite, cooling from $300 \mathrm{~K}$ to $10 \mathrm{~K}$ causes small increases in all six principal elastic constants: $C_{11}, C_{33}, C_{44}, C_{66}, C_{12}, C_{13}$. Increases vary from $2 \%$ to $10 \%$.
(2) The quantity $e_{14}^{2} / k_{11}$ involving the piezoelectric coefficient and a dielectric constant changes little with temperature.

(3) No evidence appears for a very-low-temperature structural phase transformation as appears at moderate pressures.

(4) The tetragonal-mode shear constant $C^{\prime}=\left(C_{11}-C_{12}\right) / 2$ decreases continuously, about $5 \%$ total.

(5) From our $C_{i j}$ extrapolated to zero temperature, we calculated a Debye temperature of $235 \mathrm{~K}$, close to a specific-heat value, and close to our previous estimate based on ambient-temperature $C_{i j}$.

(6) From the slope $d C^{\prime} / d T$, we obtain a negative Gruneisen parameter $\gamma\left(C^{\prime}\right)=-0.9$, agreeing well with the lowtemperature specific-heat Gruneisen parameter, $\gamma=-1$, emphasizing the dominance of $C^{\prime}$ in the low-frequency low-temperature lattice-vibration modes.

(7) The remarkable elastic anisotropy, for example $C_{66} / C^{\prime}$ $=28$, changes little with temperature. Thus, it reflects intrinsic interatomic interactions.

${ }^{1}$ H. Ogi, M. Fukunaga, M. Hirao, and H. Ledbetter, Phys. Rev. B 69, 024104 (2004).

${ }^{2}$ E. Damen, A. Aarts, and H. de Wijn, Phys. Rev. B 59, 349 (1999).

${ }^{3}$ Y. Ohmachi and N. Uchida, J. Appl. Phys. 41, 2307 (1970).

${ }^{4}$ I. Silvestrova et al., Phys. Status Solidi A 101, 437 (1987).

${ }^{5}$ H. Schweppe, Ultrasonics 8, 84 (1970).

${ }^{6}$ M. Baruci et al., J. Low Temp. Phys. 123, 303 (2001).

${ }^{7}$ L. Landau and E Lifshitz, Statistical Physics (Pergamon, Oxford, 1994), pp. 194-195.

${ }^{8}$ I. Ohno, J. Phys. Earth 24, 355 (1976).

${ }^{9}$ A. Migliori et al., Physica B 183, 1 (1993).

${ }^{10}$ A. Migliori and J. Sarrao, Resonant Ultrasound Spectroscopy (Wiley, New York, 1997).

${ }^{11}$ I. Ohno, Phys. Chem. Miner. 17, 371 (1990).

${ }^{12}$ J. Scheurer, IEEE Trans. Ultrason. Ferroelectr. Freq. Control 49, 1474 (2002).

${ }^{13}$ G. White, S. Collocott, and J. Collins, J. Phys.: Condens. Matter 2, 7715 (1990).

${ }^{14}$ G. Leibfried and W. Ludwig, Solid State Phys. 12, 275 (1961).

${ }^{15}$ Y. Varshni, Phys. Rev. B 2, 3952 (1970).

${ }^{16}$ H. Ledbetter, Phys. Status Solidi B 181, 81 (1994).

${ }^{17}$ A. Ballato, IEEE Trans. Ultrason. Ferroelectr. Freq. Control 43, 56 (1996).

${ }^{18}$ R. Lakes, Science 235, 1038 (1987).

${ }^{19}$ N. Keskar and J. Chelikowsky, Nature (London) 358, 222 (1992).

${ }^{20}$ A. Yeganeh-Hiri, D. Seidner, and J. Parise, Science 257, 650 (1992).

${ }^{21}$ H. Kimizuka, H. Kaburaki, and T. Kogure, Phys. Rev. Lett. 84, 5584 (2000).

${ }^{22}$ B. Luthi and W. Rehwald, in Structural Phase Transitions I (Springer, Berkin, 1981), p. 131, esp. Tables 4.2-4.6.

${ }^{23}$ P. Peercy and I. Fritz, Phys. Rev. Lett. 32, 466 (1974).

${ }^{24}$ H. Uwe and H. Tokumoto, Phys. Rev. B 19, 3700 (1979).

${ }^{25}$ D. Wallace, Thermodynamics of Crystals (Wiley, New York, 1972), p. 387.

${ }^{26}$ J. Champarnaud-Mesjard et al., J. Phys. Chem. Solids 61, 1499 (2000).

${ }^{27} \mathrm{M}$. Born and K. Huang, Dynamical Theory of Crystal Lattices (Oxford, London, 1954), pp. 227, 229, 262.

${ }^{28} \mathrm{H}$. Ledbetter, Handbook of Elastic Properties of Solids, Liquids, and Gases (Academic, San Diego, 2001), Vol. II, p. 57.

${ }^{29}$ A. Migliori, H. Ledbetter, D. Thoma, and T. Darling, J. Appl. Phys. 95, 2436 (2004)

${ }^{30} \mathrm{H}$. Ledbetter and H. Ogi., A study of $\mathrm{LiNbO}_{3}$ (to be published). 